\title{
Cost Efficient Scheduling Using Smart Contract Cognizant Ethereum for IoMT
}

\author{
G. Ravikumar ${ }^{1}$, K. Venkatachalam ${ }^{2}$, Mehedi Masud ${ }^{3}$ and Mohamed Abouhawwash ${ }^{4,5, *}$ \\ ${ }^{1}$ Department of Computer Science and Engineering, Coimbatore Institute of Engineering and Technology, Coimbatore, \\ 641109 , India \\ ${ }^{2}$ Department of Applied Cybernetics, Faculty of Science, University of Hradec Králové, 50003, Hradec Králové, Czech Republic \\ ${ }^{3}$ Department of Computer Science, College of Computers and Information Technology, Taif University, Taif, 21944, \\ Saudi Arabia \\ ${ }^{4}$ Department of Mathematics, Faculty of Science, Mansoura University, Mansoura, 35516, Egypt \\ ${ }^{5}$ Department of Computational Mathematics, Science, and Engineering (CMSE), Michigan State University, East Lansing, \\ MI, 48824, USA \\ *Corresponding Author: Mohamed Abouhawwash. Email: abouhaww@msu.edu \\ Received: 12 October 2021; Accepted: 15 December 2021
}

\begin{abstract}
Recently internet of medical things (IoMT) act as a smart doctor using sensor wearable's device in human body. This smart doctor device senses necessary medical data from human and transfer via network immediately to physician. It is important to transfer sensitive data very securely. Blockchain becomes trending technology to provide high security to both end users in the network. Traditionally security structure is relying on cryptographic techniques which is very expensive and takes more time in securely transmitting data. To overcome this issue, this paper builds a cost effective, blockchain with IoMT using fog-cloud computing. The aim of research is to provide cost effective healthcare services in the present system. This study develops an IoMT systems using fusion of scheduling techniques in blockchain. We propose a Smart Contract with Function Based Cost Efficient Task Scheduling (FTS-SCON) algorithm in blockchain framework. The proposed algorithm uses functions to schedule the task in the blockchain which is cost effective. With the help of cryptography based Blockchain schemes with smart contracts enables consistency and validation of data with symmetric cryptography. Simulation results shows the proposed outperform all existing elements regarding data security, validation by $10 \%$, and cost of application execution by $30 \%$ in IoMT.
\end{abstract}

Keywords: IOMT; blockchain; health care sensor; security; task scheduling algorithm; smart contract

\section{Introduction}

In recent years, Corona virus (Covid-19) caused many hazards in our society. The World Health Organization declared that Corona virus is a pandemic disease which spread all over the world. Due to this situation people suffers a lot for their general medical check-ups. To overcome this situation, IoMT is used in the medical field. Healthcare IOT is another name for Internet of Medical Things (IoMT). Many

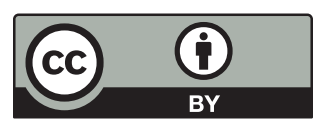

This work is licensed under a Creative Commons Attribution 4.0 International License, which permits unrestricted use, distribution, and reproduction in any medium, provided the original work is properly cited. 
biosensors were introduced and included with IOT- enabled healthcare system to support numerous applications. (i.e., Ingestible sensors, Patch sensors, Wearable, Smart clothing, Connected health sensors) $[1,2]$. The IoMT systems were used to reduce the travel time and burden of the patient and doctors. Ensuring security to the patient data is a difficult problem. Everyone is using technological devices. Costeffective and secured data transmission characteristics are a most important need for IoMT development.

State of art provides blockchain it is better source for IoMT data security [3]. Ethereum is considered as decentralized approach that helps user to initiate their own regulations and contracts. Blockchain is a secured data structure which is connected with the data blocks. It is similar to linked list with decentralized concept. Ethereum is generally used in peer-peer networks and in distributed applications.

Fog cloud computing is also known as Fog network. Fog cloud network is cooperative by which remote cloud permits visa where Fog framework presents its service to edge network. Fog computing offers distributed services on health to compute all class and the different types of applications. The three services are: 1) demands 2) reserves 3) instance at spot. It is hour, week, month, and year-based duration with various prices.

In this paper, result investigates with task scheduling techniques, cost-effectiveness, security method in the fog based blockchain at cloud environment. The main goal is to reduce cost during data scheduling and offloading. To overcome this issue, the proposed system uses the fusion of algorithm techniques such as Function Based task scheduling blockchain-enabled framework (FTSB) with blockchain-enabled costefficient smart contract for scheduling framework (FTSB-BESCAF). This study proposed the functionbased task scheduling blockchain-enable framework (FTSB), it has different schemes.

1. To solve the scheduling problem of workflow healthcare tasks with different constraints such as deadline, precedence constraint.

2. Function verification: It verifies the correctness of the function (e.g., Worms free)

3. Function Pool: It collects all the verified functions for applications and store the data in the pool

4. Priority Queue: It orders all the priority method into the application.

5. Task Scheduling: This study helps to schedule all tasks based on deadline and the reduced application costs

The goal is to decrease cost of workflow during process of scheduling in network. The smart contract in blockchain used for scheduling algorithm (BECSAF) to reduce cost framework, it has the rules as follows: smart contracts, function pool validation, function sequencing, task allocation, matching of resources, task scheduling using contracts of blockchain.

Rest of the paper is organized as sections as shown. The Section 2 consists of brief study of existing IoMT system and block chain. Section 3 explains the working principal of the proposed model. Section 4 evaluates the result and gives comparison of different algorithms. Section 5 concludes the research work with the future scope.

\section{Related Work}

IoMT is widely used in the medical field to maintain the different applications which is currently growing. To boost the performance of the IoMT network using cloud computing, it consists of network technologies, healthcare sensors and cloud computing technologies to run the various healthcare applications. The IoMT systems were used to reduce the travel time and burden of the patient and doctors. Ensuring security to the patient data is a difficult problem. Everyone is using technological devices. Cost-effective and secured data transmission characteristics are a most important need for IoMT development. In IOMT application data will encrypt for security reasons.

There are many sensors used in IoMT applications they are mobile based devices, heart sensors, pressure-based sensors, ECG and other devices connects with the mobile device [4]. Fog cloud 
computing is a network, in that cooperative network remote cloud may offers via internet, at the edge of the network cloud computing provides services. There are vast number of Medicare applications were build based on IoMT, people widely used these applications with the help of mobile based devices [5].

At the same time, sensors are communicated to servers using fog-cloud network to perform many health based tasks for patients [6]. In general, devices execute as client and fog-cloud nodes are processed in IoMT. There are so many research challenges in offloading mechanism for smart health application like malware attack, security, service denial and the storage problems [7]. Nodes in fog cloud network offer paid distributed health services to all classes [8]. Generally, these workflow applications require processes in sequential order to execute user request to next orders [9].

The service provider performs the data computation and client's requests are processed. So, the client presents key to the server for data encryption before performing the calculation appropriately. This affects the confidentiality of data [10]. Ethereum is open source decentralized blockchain which makes user to initiate the smart contracts with native rules and regulations. It is widely used in networks which are widely used in distributed application system. Also, it is collection of well-structured data blocks which cannot be unaltered and transaction logs were recorded in the blocks [11]. Each and every block has many data transactions.

The blockchain-Enable distributed for the healthcare body area network suggested. The main goal is to reduce security at end-to-end risks of applications in these studies [12]. The studies focused on failure aware mechanism in IoMT network for the healthcare applications to minimize the failure and validation risk in the system. To our knowledge, this work formulates the cost-efficient scheduling of Internet of Medical Things (IoMT) workflow tasks in function and blockchain-enable distributed network which has not been studied yet with the considered constraints.

Tab. 1 gives the detailed review on IoMT system survey. The applications cost and resources are reduced using server less IoMT system. Cost of the cloud is very low in serverless architecture. Our main objective is to identify technology with less execution cost and data offloading cost. Traditional resource functions are replaced, and multiple objectives are achieved. The objectives like reducing delay, cost, energy etc. In this proposed model is based on static optimization and solving scheduling problems using fog cloud network.

Table 1: Existing blockchain enable IoMT system and its objectives

\begin{tabular}{|c|c|c|c|c|}
\hline Study & Problems & Constraints & $\begin{array}{l}\text { Methodologies } \\
\text { used }\end{array}$ & Objective \\
\hline Lali Dootio et al. [1] & Scheduling & $\begin{array}{l}\text { Execution time, } \\
\text { cost }\end{array}$ & $\begin{array}{l}\text { Linear } \\
\text { optimization }\end{array}$ & Lateness \\
\hline Hussain et al. [3] & Offloading & $\begin{array}{l}\text { Security, } \\
\text { execution time }\end{array}$ & $\begin{array}{l}\text { Static } \\
\text { optimization }\end{array}$ & $\begin{array}{l}\text { Minimum } \\
\text { delay, risk }\end{array}$ \\
\hline Adzic et al. [7] & Offloading & Tardiness & $\begin{array}{l}\text { Static } \\
\text { optimization }\end{array}$ & $\begin{array}{l}\text { Minimum } \\
\text { delay }\end{array}$ \\
\hline Lynn et al. [8] & Allocation & Tardiness & $\begin{array}{l}\text { Static } \\
\text { optimization }\end{array}$ & $\begin{array}{l}\text { Minimum } \\
\text { tardiness }\end{array}$ \\
\hline VanEyk et al. [9] & Resource & Lateness & $\begin{array}{l}\text { Static } \\
\text { optimization }\end{array}$ & $\begin{array}{l}\text { Minimum } \\
\text { Tardiness }\end{array}$ \\
\hline $\begin{array}{l}\text { Perez et al. [10] and } \\
\text { Lara et al. [11] }\end{array}$ & Scheduling & $\begin{array}{l}\text { Execution time, } \\
\text { cost }\end{array}$ & $\begin{array}{l}\text { Linear } \\
\text { optimization }\end{array}$ & Lateness \\
\hline $\begin{array}{l}\text { Lara et al. [11], Lakhan et al. [12] } \\
\text { and Xiaoping et al. [13] }\end{array}$ & Scheduling & $\begin{array}{l}\text { Execution time, } \\
\text { cost }\end{array}$ & $\begin{array}{l}\text { Dynamic } \\
\text { optimization }\end{array}$ & Scale-up \\
\hline
\end{tabular}




\begin{tabular}{|c|c|c|c|c|}
\hline Study & Problems & Constraints & $\begin{array}{l}\text { Methodologies } \\
\text { used }\end{array}$ & Objective \\
\hline Lakhan et al. [14] & Offloading & Security & $\begin{array}{l}\text { Dynamic } \\
\text { optimization }\end{array}$ & Minimum risk \\
\hline Wah et al. [15] & $\begin{array}{l}\text { Task } \\
\text { allocation }\end{array}$ & Security & $\begin{array}{l}\text { Dynamic } \\
\text { optimization }\end{array}$ & Minimum risk \\
\hline Khoso et al. [16] & $\begin{array}{l}\text { Task } \\
\text { allocation }\end{array}$ & Security & $\begin{array}{l}\text { Dynamic } \\
\text { optimization }\end{array}$ & Minimum risk \\
\hline
\end{tabular}

\section{Proposed FTS-SCON Methodology for IOMT}

\subsection{Overview}

The major challenge in IoMT system is insecure transactions and cost effective. In the existing systems, Function Based Task scheduling Blockchain Enable Framework method is used in blockchain enabled IoMT, to reduce the workflow task during scheduling and process in the network. This research combines both the Task Scheduling method and Cost-Effective method (FTS-SCON) to minimize the cost and workflow task during scheduling process. The FTS-SCON algorithm gives

- To schedule all the tasks in the way and trade-off Between Function cost and deadline of the task.

- It minimizes the cost of the healthcare application services to the network.

\subsection{Function Based Task Scheduling Framework}

Scheduling in cloud computing is an NP_hard optimization problem. Security is the most important task scheduling. Some of the tasks may need privacy policies and data protection. FTSB which consist of different schemes: Function Verification, Function Pool Priority Queue Scheduling. This framework is to schedule all the tasks in the way and trade-off between deadline of the task and cost of the function.

\subsection{Cost Efficient Scheduling Scheme using Smart Contract}

The major need is to reduce the cost during data offloading and scheduling application in the blockchain at the fog and the cloud network. A Deadline is provided to each task to complete with the threshold in the workflow. The cost-efficient scheduling is solved using IoMT application, the BECSAF consists of following schemes:

- Smart-Contract rule selects each node connects in the IoMT system; there is a different execution cost in each function.

- Function Verification Pool method identifies the security requirement of functions before adding to the system pool.

- Tasks Sequencing and Functions Sequencing

- Matching of resources

\subsection{System Model}

The system model shows the general architecture and workflow of algorithm in the system. Fig. 1 represents each and every stage of workflow. It has several stages they are explained below: 


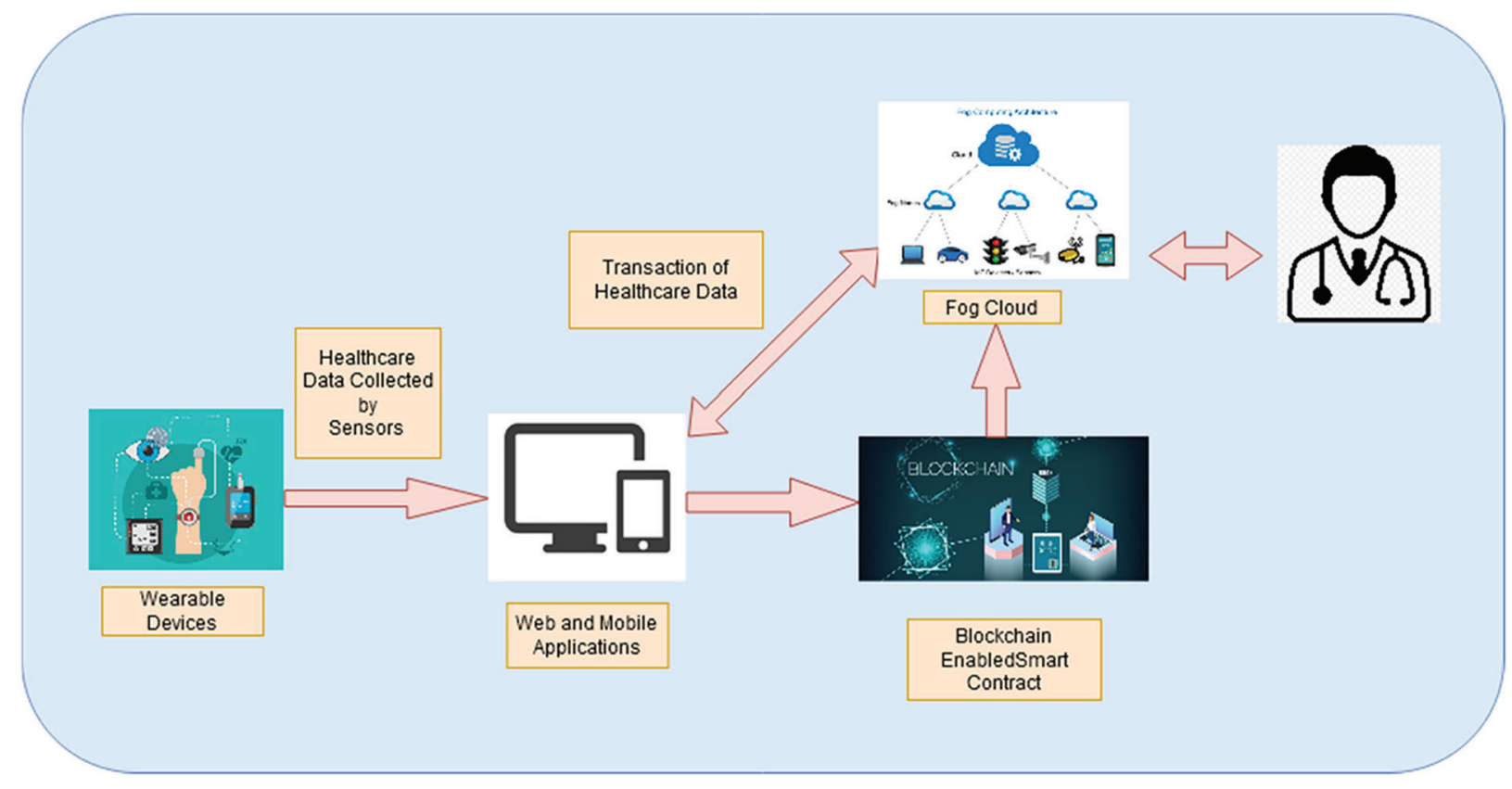

Figure 1: Overall architecture of FTS-SCON

Wearable devices are smart electronic devices, which helps to detect, analyze, and transmit information through healthcare application services. Wearable devices such as optimizer, smart belts, chest straps etc. Healthcare sensors were directly stored the data in blockchain enabled smart contract and fog cloud servers to perform several tasks. From the fog cloud server, it collects all the data and transmits to the physician. Server in Fog cloud stores all the healthcare data it has fog-cloud nodes, which assist to process all data request in the IoMT system. Fog based cloud computing offers may healthcare services to process all classes and the application.

\subsection{Blockchain Enabled Smart Contract}

Ethereum is an open source and decentralized blockchain, which allows user to create smart contracts. Ethereum blockchain contains group of data blocks, they are well structured and unalterable also it records all the transactions in the files. Data blocks stores it data in node. Environment of blockchain performs several investigations in providing security for medical data. Smart contracts are used for accessing control, which helps to detect and tracks the data sharing to trespasser.

\subsection{Proposed Method of FTS-SCON Algorithm}

The proposed system is shown in Fig. 2 consists of different techniques. The IoMT healthcare system consists of workflows that connect several sensors and offload the tasks to the Blockchain enable Fog Cloud Healthcare Servers for execution. 


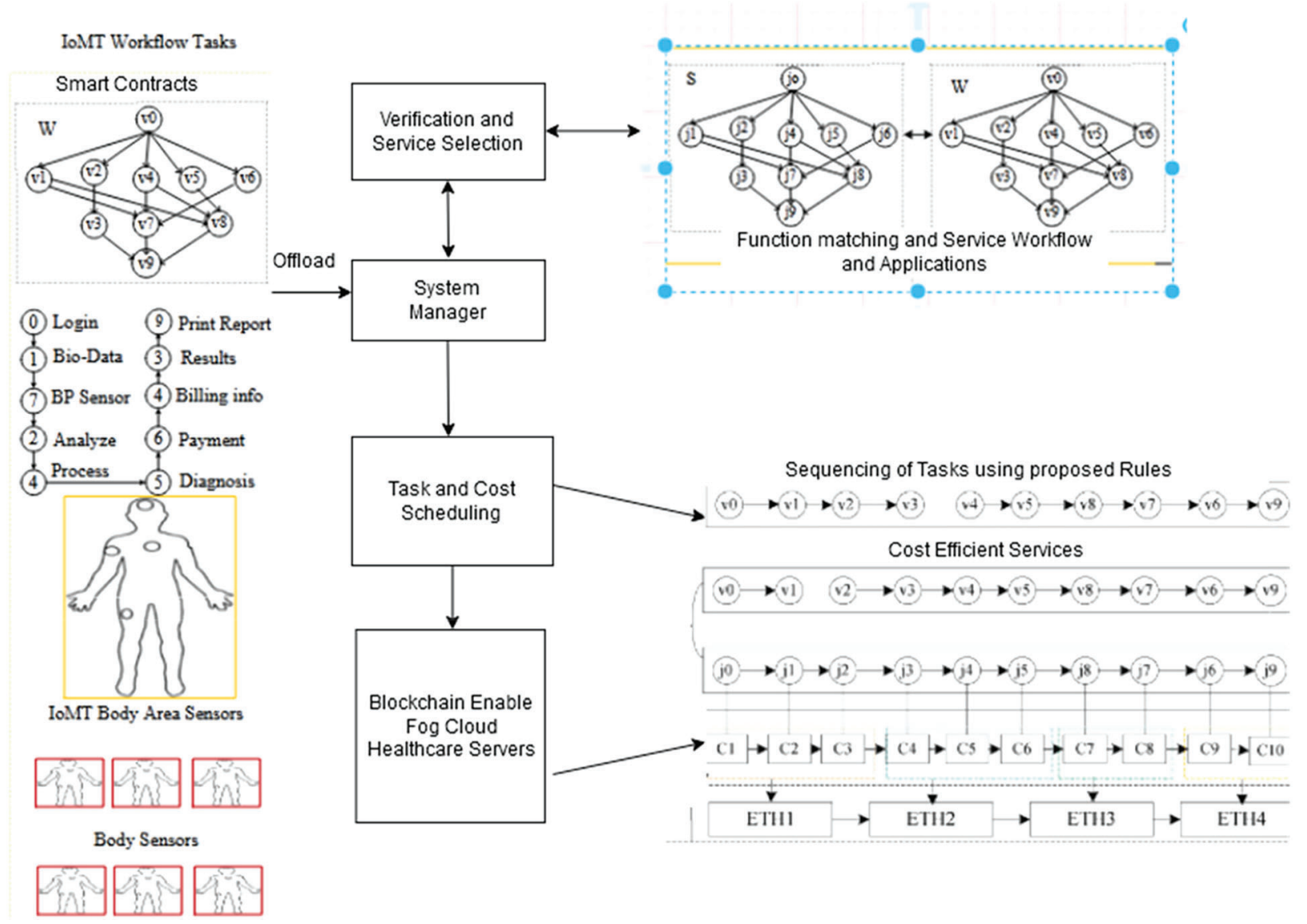

Figure 2: Task and cost scheduling method and smart contract enabled blockchain network for IoMT

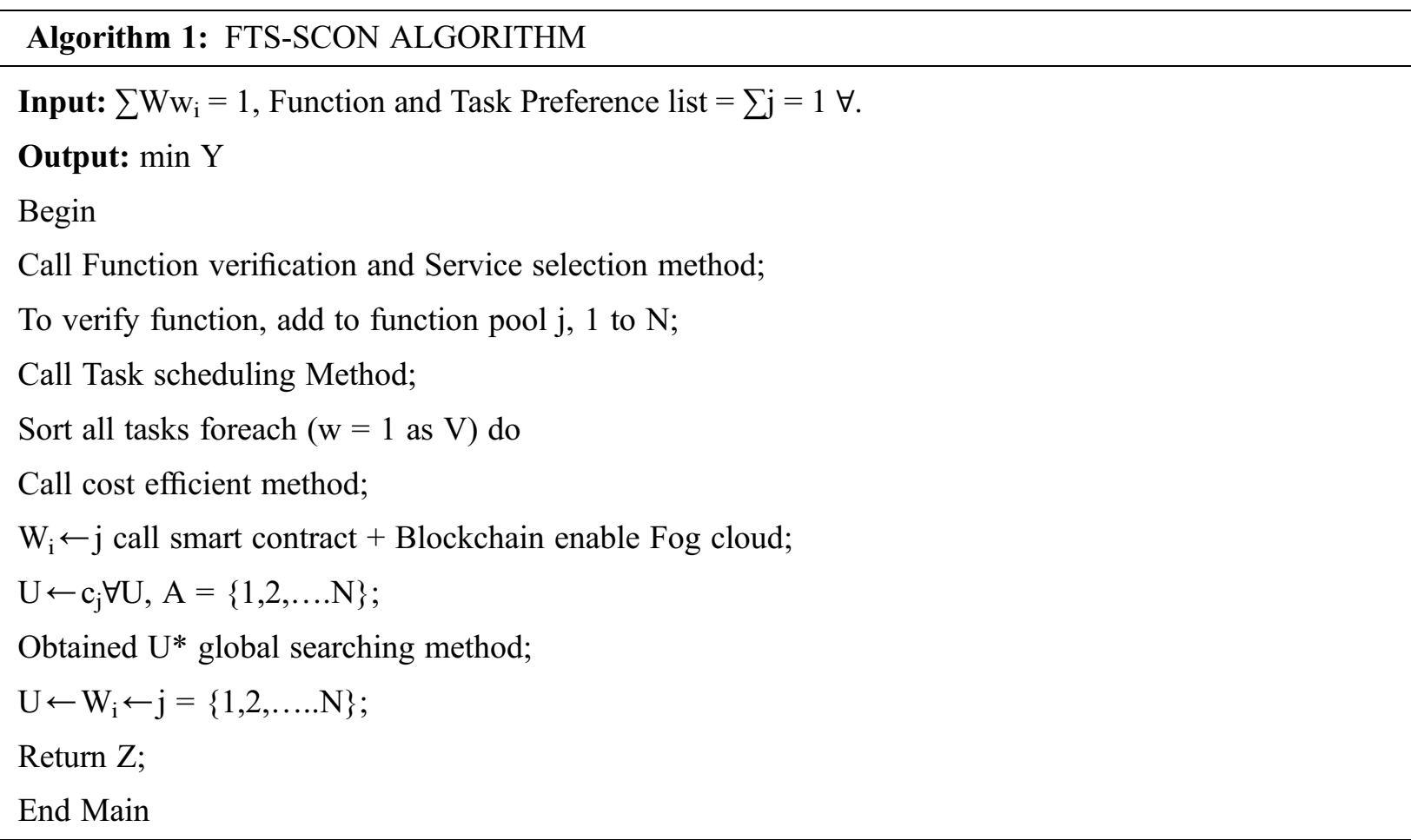




\subsection{Function Verification and Service Selection}

The Service selection and verification is a method; thus, healthcare services are added to the service pool and the transaction is verified using blockchain technique. Cloud providers like AWS Lambda, Azure, Google cloud, IBM, Alibaba Function compute offers many healthcare services. The workflow application of Quality of Service (QoS) such as deadline, to run task data should be optimal in the system.

The function verification method identifies the security requirement of functions before adding functions to the system pool. These systems are distributed and self-replicated. The malware has some capacity to be labelled as a worm or virus. Many Fog healthcare servers also connected with them. The transactions are verified/ validated by the device connected in the network. There are certain rules set by developers in the blockchain network. Once the transaction is validated in the block, it is sealed by a lock using (hash). Now the transaction is the part of blockchain.

\subsection{Task Scheduling Algorithm}

Novel service composition method determines the match of each function to each task before scheduling. Algorithm 1 takes task and prefers function as input. Based on requirements of costs and tasks, match list was created, where each task is to assign to a function which satisfies its requirements. It matches all the tasks in the end till the list is empty.

Algorithm 2: Task and Cost Scheduling Algorithm

Input: $\sum W w i=1$, Function Preference List $\sum \mathrm{j}=1$ Task preference List $\sum \mathrm{Yy}=1$;

Output: $\min X$;

Begin

Foreach ( $\mathrm{j}-1$ as $\mathrm{W})$ do

Foreach ( $\mathrm{y}-1$ as $\mathrm{Y})$ do

While (Tijk $\neq$ empty) do

Search best Tjik is picked for yi;

Compare each timeslot of service for each task Tjik in Wij;

If (Tjik in Wij is matched) then

Calculate $\mathrm{S} \leftarrow[\mathrm{xjik}]$;

Add Match $[\mathrm{Sij} \leftarrow \mathrm{xjik}]$;

Match [Sij,xjik];

End Main

According to task sequencing and composition matching list, the scheduler allocates each task to the function based on requirements. The composition list of tasks and functions, schedule the task based on its cost ad deadline. Until every task gets allocated and executed to appropriate functions, it iteratively carries the process. 


\subsection{Client Side-Smart Contract Mechanism in Fog-Cloud}

Fig. 3 defines each and every task based on data requirement, data $\mathrm{i}, \mathrm{z}$ for executing in various nodes, e.g., $\mathrm{k}=1$.

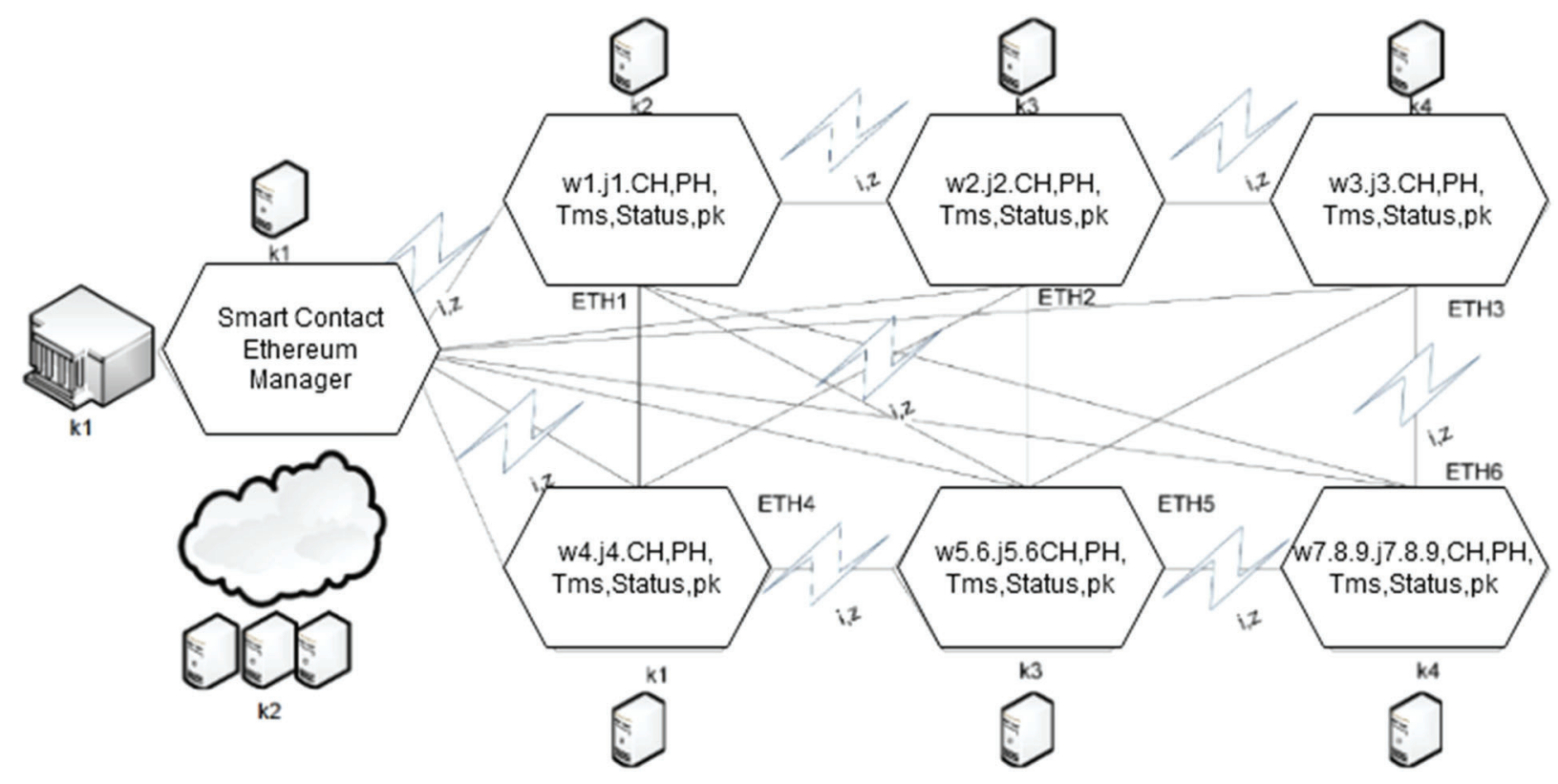

Figure 3: Smart contract Ethereum mechanism in client fog cloud

- A node executes a transaction process for all tasks based on smart contracts, that the data encryption is done using public key in blockchain.

- The data sharing between each task wi and wz should be validated by proof of work protocol.

Each and every Ethereum performs hashing technique based on public key. Fig. 3 shows the Ethereum transaction of particular node, by using the functions inside the container.

\section{Result and Evaluation}

\subsection{System Implementation}

The Functions as a Service (FaaS) is considered as a serverless system mainly designed for evaluating the performance of the system based on various workloads. Fig. 4 shows the implemented components. Blockchain is executed inside the system, for maintaining the distributed environment. The system is already designed and defined in the previous study. The two vendors function such as IBM Open Whisk and Azure were added into the system. The main aim is to execute all the task on functions using blockchain network. Ethereum blockchain ETH and Smart-Contract were implemented in Fig. 4.

\subsubsection{IoMT Sensors}

The Sensor used for knowing heartbeat is used for measuring rate of heart i.e., velocity of Heart. Heart rate, temperature and pressure are the basic health data we to do keep our self-safe. Thermometer is used for calculating the body temperature and sphygmomanometer is used for monitoring the blood pressure/arterial pressure. There are two possible ways to track the heart rate. First one is a manual checkup and the second one is heartbeat sensors. In this research, we use Arduino Heart Rate Monitor and Heartbeat Sensor. 
Heartbeat control is very essential for patients and athletes. Today smart wristwatch, smart phones etc., are available for sensing heartbeat. The measure of heart beats is represented as beats per minute or bpm.

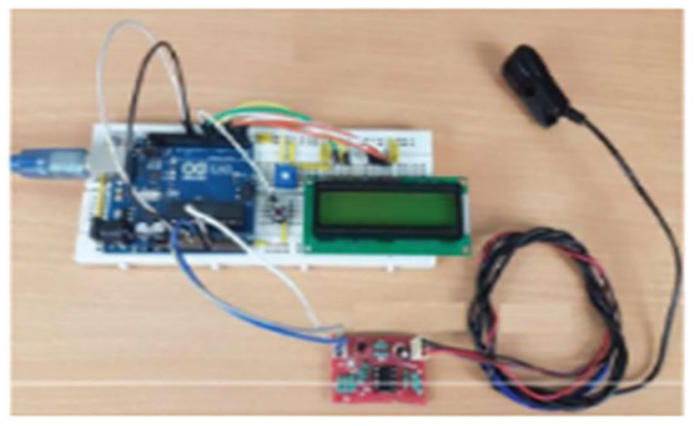

(a) Arduino Heartbeat Board

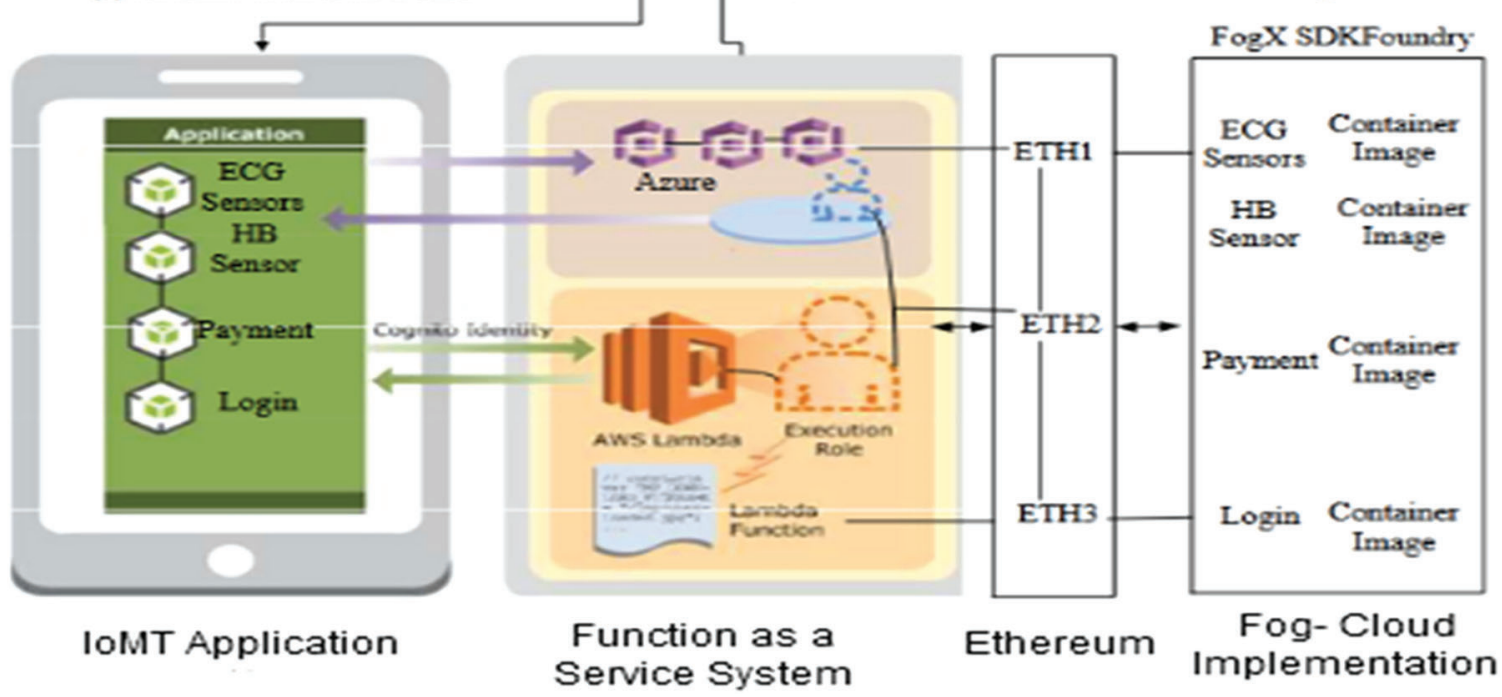

Figure 4: Smart contract ethereum cognizant fog cloud system for IoMT

\subsubsection{IoMT Application}

IoMT application consists of four sub applications in it: 1) cancer-awareness monitoring, 2) Heartbeat, 3) ECG, 4) EEG monitoring. Fig. 4 shows workflow tasks and various functions are needed to run.

\subsection{Baseline and Function Verification Approaches}

The performance of existing and proposed systems was evaluated the system based on execution of resources and applications on deadline and cost. Existing studies [17-26] suggested blockchain-enabled fog-cloud network for healthcare applications. The study recommends the method for identifying the standard function it should be cleared from any security issues to any issues.

\subsection{Result Discussion}

The functions and their characteristics determine the cost of application execution. All functions have various computation time and memory. So, the computation cost of each and every function has a different execution time and memory. Fig. 5 shows the existing system, it is hard to maintain the deadlines during scheduling, and balance the execution cost. Also, there is a security risk. 

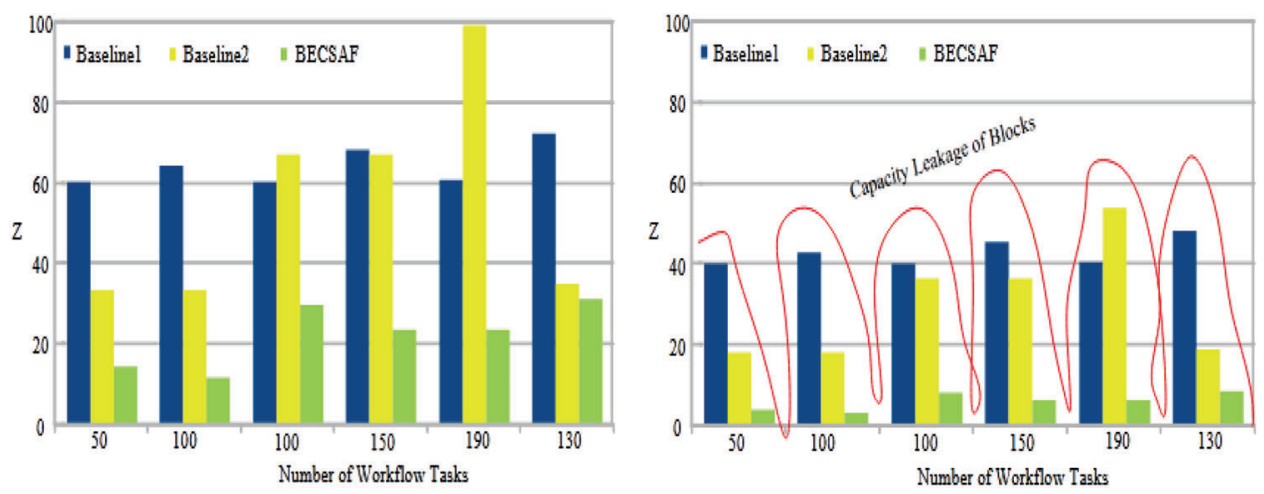

Figure 5: Existing proof of sake [1] for IoMT workflow transactions in blockchain enabled

\subsubsection{Fog-Cloud Network and Resource Capacity Leakage of Blocks in Blockchain}

Comparing with the existing system, the proposed system achieves the reduction of cost in the healthcare application servers. Fig. 6 shows the comparison of existing algorithms FTSB and BECSAF with the proposed algorithm FTS-SCON. The accuracy of the proposed system performs all the tasks and run in the Fog-cloud servers. It gives more accuracy than the existing system during implementation. Each methods have different workflow tasks, comparing with the existing system the computation time is better in the proposed system. The FTS-SCON method has more computation time.

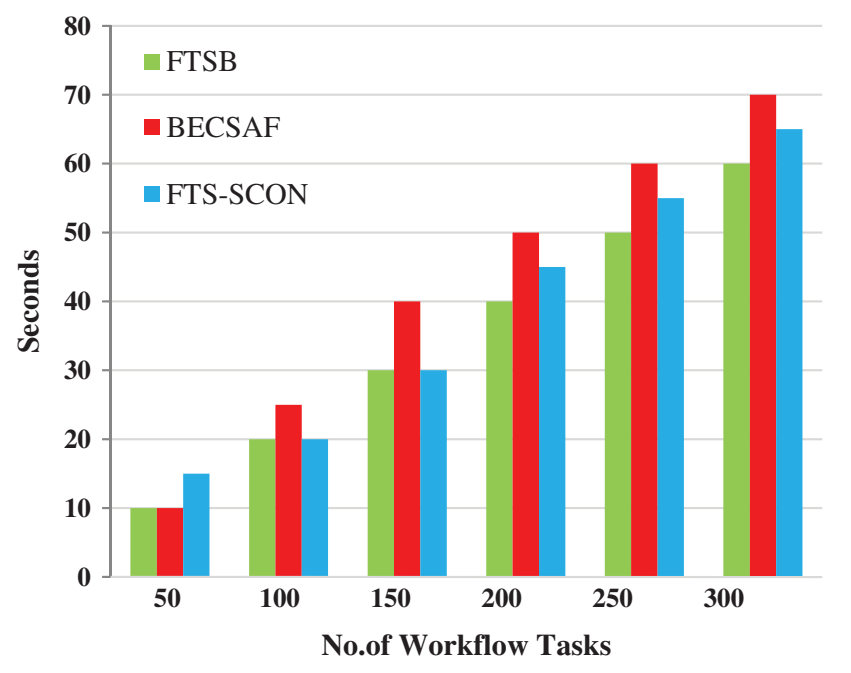

Figure 6: Performance analysis of task scheduling algorithm

\subsubsection{Cost Scheduling Algorithm}

In the existing system, use FTSB algorithm for reducing the cost of the healthcare system. The existing system has some security issues while running the task, this causes a data loss. Fig. 7 shows the relative deviation percentage (RDP\%). performance takes (memory*execution) for the workflow of healthcare system. There are two baseline methods were used in the existing system. In the proposed system, along with FTSB, BECSAF and FTS-SCON algorithms were compared. By using these algorithms, it provides the execution cost of the healthcare system is reduced. Fig. 8 shows the cost-efficient scheduling analysis.

The accuracy level of FTS-SCON is higher than the existing system. It also works with the baseline, in the baseline 1 exploits the static resource provisioning cost. 


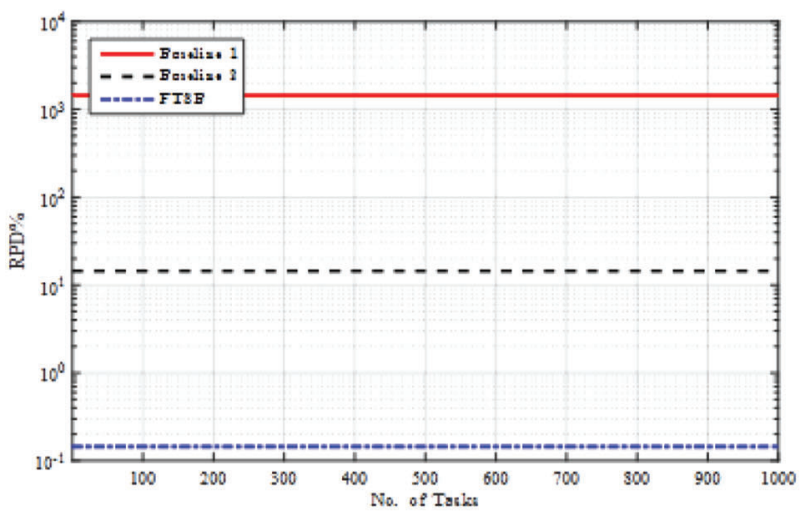

(a)

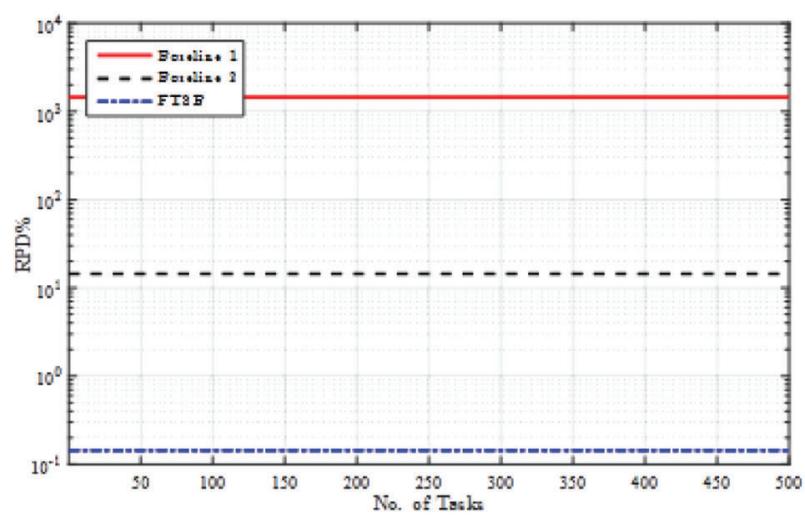

(b)

Figure 7: Existing cost-efficient scheduling [2] (a) Initial cost of functions (b) After $30 \mathrm{~s}$ interval the cost of functions

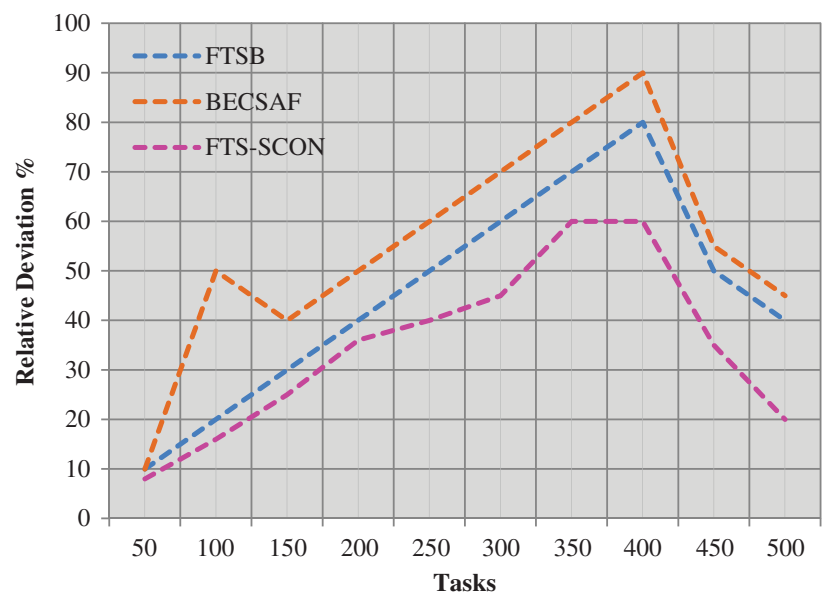

Figure 8: Performance analysis of cost-scheduling algorithm

Baseline 2 performs the dynamic resource provisioning. The proposed FTS-SCON, Fig. 8 outperforms all the tasks in terms of cost. It reduces the security risks because the data is stored in the Fog-Cloud enabled Ethereum. Thus, Ethereum contains several blocks, each block is connected so the healthcare information becomes safe. The verification method validates all the tasks. It uses (memory*execution) i.e., 500*1000. FTS-SCON achieves more results.

\section{Conclusions}

The research develops the novel, cost efficient service selection and secured the IoMT with Ethereum using Blockchain in Fog Cloud for healthcare applications. The main objective is to decrease the healthcare cost in service applications system and to reduce the risk at security functions while implementing system pool. IoMT system used healthcare system with the condition of security and cost in the medical system showed in result evaluation. The proposed FTS-SCON algorithm works along with the metrics, it minimizes the cost of the healthcare service applications, and it minimizes the security risks. Thus, the algorithm is more effective while implemented in the IoMT systems. 
In future scope of the work, this research will extend the IoMT system with Deep learning techniques for the health-based applications i.e., Drone systems. Serverless systems may reduce the cost of the applications. By using serverless systems, the results may transfer to the cloud systems based on mobility and security will be suggested for future improvement in the field of healthcare systems.

Acknowledgement: We would like to give special thanks to Taif University Researchers supporting Project Number (TURSP-2020/10), Taif University, Taif, Saudi Arabia.

Funding Statement: Taif University Researchers supporting Project Number (TURSP-2020/10), Taif University, Taif, Saudi Arabia.

Conflicts of Interest: The authors declare that they have no conflicts of interest to report regarding the present study.

\section{References}

[1] A. Lali Dootio, A. HSodhro, S. Pirbhulal, T. Morten, M. Saddam et al., "Cost-efficiet service selection and execution and blockchain-enables serverless network for internet of medical things," Mathematical Biosciences and Engineering, vol. 18, no. 3, pp. 18-24, 2021.

[2] A. Lakhan, M. A. Mohammed, A. N. Rashid, S. Kadry, T. Panityakul et al., "Smart-contract aware ethereum and client-fog-cloud healthcare system," Sensors, vol. 21, no. 12, pp. 24-35, 2021.

[3] M. Hussain, L. F. Wei, A. Lakhan, S. Wali, S. Ali et al., "Energy and performance-efficient task scheduling in heterogeneous virtualized cloud computing," Sustainable Computing, Informatics and Systems, vol. 30, no. 4, pp. 1-13, 2021.

[4] A. Lakhan, M. Ahmad, M. Bilal, A. Jolfaei and R. M. Mehmood, "Mobility aware blockchain enabled offloading and scheduling in vehicular fog cloud computing," IEEE Transactions on Intelligent Transportation Systems, vol. 22, no. 7, pp. 4212-4223, 2021.

[5] T. Ying Wah, R. Gopal Raj and A. Lakhan, "A novel cost-efficient framework for critical heartbeat task scheduling using the internet of medical things in a fog cloud system," Sensors, vol. 20, no. 2, pp. 1-12, 2020.

[6] F. H. Khoso, A. Lakhan, A. Arain, M. A. Soomro, S. Z. Nizamani et al., "A microservice-based system for industrial internet of things in fog-cloud assisted network," Engineering, Technology and Applied Science Research, vol. 11, no. 2, pp. 7029-7032, 2021.

[7] G. Adzic and R. Chatley, "Serverless computing: Economic and architectural impact," in Proc. European Software Engineering Conf. (ESEC), Paderborn, Germany, pp. 884-889, 2017.

[8] T. Lynn, P. Rosati, A. Lejeune and V. Emeakaroha, "A preliminary review of enterprise serverless cloud computing (function-as-a-service) platforms," in Proc. Int. Conf. on Communication and Computational Technologies (ICCCTS), Hong Kong, China, pp. 162-169, 2017.

[9] E. VanEyk, A. Iosup, S. Seif and M. Thömmes, "The SPEC cloud group's research vision on FaaS and serverless architectures," in Proc. Int. Wine and Spirit Competition (IWSC), Las Vegas, USA, pp. 11-15, 2017.

[10] A. Pérez, G. Moltó, M. Caballer and A. Calatrava, "Serverless computing for container-based architectures," Future Generation Computer Systems, vol. 83, no. 4, pp. 50-59, 2018.

[11] D. Lara, E. Gomes, C. S. Langridge, S. Mortazavi and S. H. Roodi, "Hierarchical serverless computing for the mobile edge," in Proc. Institute for Social and Economic Change (ISEC), Washington, USA, pp. 109-110, 2016.

[12] A. Lakhan and X. Li, "Transient fault aware application partitioning computational offloading algorithm in microservices based mobile cloudlet networks," Computing, vol. 102, no. 1, pp. 105-139, 2020.

[13] A. Lakhan and L. Xiaoping, "Energy aware dynamic workflow application partitioning and task scheduling in heterogeneous mobile cloud network," in Int. Conf. on Computational Biology and Bioinformatics (ICCBB), Fuzhou, China, pp. 1-8, 2018. 
[14] A. Lakhan and X. Li, "Content aware task scheduling framework for mobile workflow applications in heterogeneous mobile-edge-cloud paradigms: CATSA Framework," in Proc. Int. Society for the Performing Arts (ISPA), Xiamen, China, pp. 242-249, 2019.

[15] T. Wah, R. Gopal Raj, A. Lakhan and Q. Mastoi, "A novel cost-efficient framework for critical heartbeat task scheduling using the internet of medical things in a fog cloud system," Sensors, vol. 20, no. 2, pp. 441, 2020.

[16] F. Khoso, A. Lakhan, A. Arain, M. Soomro, S. Nizamani et al., "A microservice based system for industrial internet of things in fog cloud assisted network," Engineering, Technology \& Applied Science Research, vol. 11, no. 2, pp. 7029-7032, 2021.

[17] N. Islam, Y. Faheem, U. Din, M. Talha, M. Guizani et al., "A blockchain-based fog computing framework for activity recognition as an application to e-Healthcare services," Future Generation Computer Systems, vol. 100 , no. 4, pp. 569-578, 2019.

[18] M. Abdel-Basset, N. Moustafa, R. Mohamed, O. Elkomy and M. Abouhawwash, "Multi-objective task scheduling approach for fog computing," IEEE Access, vol. 9, no. 14, pp. 126988-127009, 2021.

[19] M. Waseem, A. Lakhan and I. Jamali, "Data security of mobile cloud computing on cloud server," Open Access Library Journal, vol. 3, no. 4, pp. 35-45, 2016.

[20] M. Abouhawwash and A. Alessio, "Multi-objective evolutionary algorithm for PET image reconstruction: Concept," IEEE Transactions on Medical Imaging, vol. 12, no. 4, pp. 1-10, 2021.

[21] S. Tanwar, K. Parekh and R. Evans, "Blockchain-based electronic healthcare record system for healthcare 4.0 applications," Journal of Information Security and Applications, vol. 50, no. 1, pp. 2407, 2020.

[22] F. Khoso, A. Arain, A. Lakhan, A. Kehar and S. Nizamani, "Proposing a novel IoT framework by identifying security and privacy issues in fog cloud services network," International Journal Speech Technology, vol. 9, pp. 592-596, 2021.

[23] M. Masud, G. Gaba, K. Choudhary, M. Hossain, M. Alhamid et al., "Lightweight and anonymity-preserving user authentication scheme for IoT-based healthcare," IEEE Internet of Things Journal, vol. 24, no. 2, pp. 1-12, 2021.

[24] A. Ali, H. A. Rahim, J. Ali, M. F. Pasha, M. Masud et al., "A novel secure blockchain framework for accessing electronic health records using multiple certificate authority," Applied Sciences, vol. 11, no. 21, pp. 1-14, 2021.

[25] M. Masud, G. S. Gaba, K. Choudhary, R. Alroobaea and M. S. Hossain, "A robust and lightweight secure access scheme for cloud based E-healthcare services," Peer-to-Peer Networking and Applications, vol. 14, no. 3, pp. 3043-3057, 2021.

[26] M. Masud, G. Gaba, S. Alqahtani, G. Muhammad, B. Gupta et al., "A lightweight and robust secure key establishment protocol for internet of medical things in COVID-19 patients care," IEEE Internet of Things Journal, vol. 8, no. 21, pp. 15694-15703, 2021. 